\title{
Main Line Fault Localization Methodology in Smart Grid - Part 3: Main Line Fault Localization Methodology (MLFLM)
}

\author{
Athanasios G. Lazaropoulos ${ }^{1}$ \\ 1: School of Electrical and Computer Engineering / National Technical University of Athens / \\ 9 Iroon Polytechniou Street / Zografou, GR 15780
}

Received June 13, 2017; Accepted September 2, 2017; Published October 6, 2017

\begin{abstract}
Since the main distribution line faults can be securely identified as outlined in the first and second paper, this third paper presents the methodology of localizing the main distribution line fault when broadband over power lines (BPL) networks have already been deployed across the distribution power grids. The main issue of this paper is the detailed presentation of the main line localization methodology (MLFLM) as well as well as its performance assessment when measurement differences occur.

The contribution of this paper, which is focused on the application of MLFLM, is double. First, the procedure, which is followed in order to create the database of faults and is used by MLFLM, is here analytically presented. This database is based on the application of the main distribution line fault identification percentage metric (MDLFI) to coupling reflection coefficients of all possible fault OV MV BPL topologies (modified OV MV BPL topologies). Second, the performance assessment of MLFLM is investigated with respect to the nature of the measurement differences and the location of main distribution line faults across the distribution power grid.
\end{abstract}

Keywords: Smart Grid; Intelligent Energy Systems; Broadband over Power Lines (BPL) Networks; Power Line Communications (PLC); Faults; Fault Analysis; Fault Localization; Distribution Power Grids

\section{Introduction}

A major advantage of the BPL networks is the fact that their development is simple and economically advantageous since their deployment is based on the already operating power grid infrastructure. As concerns the potential of the smart grid operation, BPL technology can act as either an autonomous communications system or a cooperative communications network that interoperates with other wired and wireless communications solutions in an integrated intelligent IP-based network environment. Anyway, the synergy of telecommunications solution under the aegis of the smart grid can support a myriad of smart grid applications [1]-[4].

In the first paper [5], the determination of the channel attenuation and reflection coefficient of overhead medium-voltage (OV MV) BPL networks have been achieved by extending the original TM2 method, which is the core part of the top-down approach of the well-established hybrid method [3], [6]-[22] during the normal operation of OV MV 
BPL networks, to the extended TM2 method. In fact, extended TM2 method can give as output the reflection coefficients during the operation of OV MV BPL networks where a main distribution line fault occurs (fault operation). In the second paper [23], the role of measurement differences that occur during the determination of reflection coefficients due to the "real-life" conditions and during the identification of main distribution line faults has been analyzed. Actually, the destructive impact of measurement differences during the main distribution line identification has been significantly counteracted through the application of piecewise monotonic data approximations (PMAs), such as L1PMA. In the vast majority of the cases examined, the fault operation has been identified by applying the main distribution line fault identification percentage metric (MDLFI), which accompanies the main distribution fault identification methodology. In this paper, the detailed presentation of the main line localization methodology (MLFLM) is provided. It is obvious that the MLFLM commences to operate since the identification of a main distribution line fault is granted on the basis of the findings of the first and second paper. Initially, MLFLM supports the preparation of a detailed database of coupling reflection coefficients and respective MDLFIs for all the possible OV MV BPL topologies. Apart from the plethora of OV MV BPL topologies that may be supported, this database also takes into account the nature of the main distribution line fault (i.e., short- or open-circuit termination load, see [5]). Then, statistics regarding the database access as well as the exact procedure that is followed in order to parse the database and, finally, to exactly localize the main distribution line fault is provided. Here, it should be noted that the previous experience concerning the creation and management of databases, which has been obtained from the Topology Identification Methodology (TIM) of [22], [24] and Fault and Instability Identification Methodology (FIIM) of [25], [26], is exploited in MLFLM.

The rest of this paper is organized as follows: In Sec.II, the findings of [5], [23] that are used in this paper are briefly outlined. In Sec.III, the creation procedure of the MLFLM database is detailed as well as implementation details and characteristics concerning its access during the fault operation. Sec.IV discusses the simulations of various OV MV BPL topologies with random main distribution line faults intending to mark out the performance efficiency of the proposed MLFLM when measurement differences occur. Sec.V concludes this paper.

\section{Brief Presentation of the OV MV BPL Topologies, Main Distribution Line Faults, Measurement Differences and MDLFI}

In accordance with [5], [23], four indicative OV MV BPL topologies of average path length of $1000 \mathrm{~m}$, which are treated as the original topologies, are also examined in this paper, namely:

1. A typical urban topology (denoted as urban case) with $N=3$ branches ( $L_{1}=500 \mathrm{~m}, L_{2}=200 \mathrm{~m}, L_{3}=100 \mathrm{~m}, L_{4}=200 \mathrm{~m}, L_{\mathrm{b} 1}=8 \mathrm{~m}, L_{\mathrm{b} 2}=13 \mathrm{~m}, L_{\mathrm{b} 3}=10 \mathrm{~m}$ ).

2. A typical suburban topology (denoted as suburban case) with $N=2$ branches ( $L_{1}=500 \mathrm{~m}, L_{2}=400 \mathrm{~m}, L_{3}=100 \mathrm{~m}, L_{\mathrm{bl}}=50 \mathrm{~m}, L_{\mathrm{b} 2}=10 \mathrm{~m}$ ).

3. A typical rural topology (denoted as rural case) with only $N=1$ branch $\left(L_{1}=600 \mathrm{~m}, L_{2}=400 \mathrm{~m}, L_{\mathrm{b} 1}=300 \mathrm{~m}\right)$.

4. The "LOS" transmission along the same end-to-end distance $L=L_{1}+\ldots+L_{N+1}=1000 \mathrm{~m}$ (denoted as "LOS" case) when no branches are 
encountered. This topology corresponds to Line of Sight transmission in wireless channels.

Already been mentioned, critical problematic conditions, such as the main distribution line faults that are examined in these three papers, can occur across the distribution power grid during its operation. With reference to Fig. 1(b) of [5], let the main distribution line be broken at a specific location from the transmitting end. On the basis of the original OV MV BPL networks, new OV MV BPL topologies occur with path lengths equal or shorter than $1000 \mathrm{~m}$ whose path lengths depend on the location of the main distribution line fault. Not only the path length but depending on the fault location, the new OV MV BPL topologies are characterized by different number of branches in comparison with the respective original ones. These new OV MV BPL topologies are denoted as modified OV MV BPL topologies. Except for their topological characteristics concerning the path length and the number of branches, modified OV MV BPL topologies differ from the respective original ones in the nature of the terminal load, which is situated at the location of the fault. While original OV MV BPL topologies are characterized by terminal loads that are matched to the characteristic impedances of the modes examined, the terminal loads of the respective modified OV MV BPL topologies are equal to either short- or open-circuit depending on the nature of the main distribution line fault (see [5] for further details). Hence, two sets of the four modified OV MV BPL topologies are examined in this subsection, each one corresponding to the two different values of the terminal load.

As been presented in [5], a main distribution line fault can be easily identified through the extended TM2 method and its coupling reflection coefficients. However, apart from the main distribution line fault, a set of practical reasons and "real-life" conditions create significant differences between experimental measurements and theoretical results. In accordance with [22], the causes of the measurement differences presented to the coupling reflection coefficients can be grouped into six categories, namely: (i) Isolation difficulties of specific MTL parameters in time- and frequencydomain; (ii) Low accuracy and sensitivity of the used equipment during measurements; (iii) Cross-talk and resonant phenomena due to the parasitic capacitances and inductances of lines; (iv) The weakness of including specific wiring and grounding practices; (v) Practical impedance deviations of lines, branches, terminations and transmitting/receiving ends; and (vi) The isolation lack of the noise effect during the transfer function computations.

The PMA experience, which has been acquired either in the case of coupling transfer functions [3], [22], [24]-[26] or in the case of coupling reflection coefficients [5], secures the identification of a main distribution line fault despite the measurement differences. On the basis of PMA piecewise monotonicity property, PMAs receive as inputs the measured coupling reflection coefficient data, the measurement frequencies and the number of monotonic sections and give as outputs the optimal primary extrema and the best fit of the measured OV MV BPL reflection coefficient data. In order to assess at the same time the mitigation performance against measurement differences and the main distribution line fault identification efficiency, the main distribution line fault identification percentage metric (MDLFI) has been proposed in [5]. MDLFI acts as the accompanying performance metric of the identification process of main distribution line faults and describes the relative error between L1PMA approximations of the measured and theoretical data. According to the results of [5], the main distribution line fault identification efficiency depends on the magnitude of the measurement differences, 
critical thresholds of the MDLFI (MDLFI ${ }_{\text {thr }}$ ) and the relative distance of main distribution line fault from the transmitting end. However, MLFLM achieves to exactly localize the main distribution line faults getting rid of the MDLFI dependencies, such as MDLFI $_{\text {thr }}$ and the relative fault distance from the transmitting end, by wisely: (i) applying MDLFI equation between coupling reflection coefficient measurements and theoretical coupling reflection coefficient data of MLFLM database; and (ii) using both the available coupling reflection coefficient measurement sites, which are the transmitting and receiving end of the examined original OV MV BPL topology (see more details in Sec.III).

\section{OV MV BPL Topology Database with MDLFI and MLFLM}

In accordance with [3], [22], the first step of MLFLM is the creation of the MLFLM database. In fact, MLFLM database consists of all possible modified OV MV BPL topologies that can occur due to main distribution line faults.

In [23], it has been identified that the identification efficiency of a main distribution line fault significantly depends on the location of the fault across the OV MV BPL topology. In fact, when the fault is located near to the transmitting end the identification becomes easier in comparison with the faults that are closer to the receiving end. In order to exploit this finding, the complimentary OV MV BPL topology to the original one from the side of the receiving end should also been considered in the database for given OV MV BPL topology. Hence, the computational load of MLFLM database becomes double in comparison with the respective one of TIM and FIIM since each database record now consists of a pair of two modified OV MV BPL topologies [3], [22], [25], [26]. Here, it should be noted that the nature of the termination load remains the same for given database record pair. Since the average transmission length is equal to $1000 \mathrm{~m}$ and the length spacing $L_{\mathrm{s}}$ that defines the database accuracy is assumed equal to $50 \mathrm{~m}$ in this paper, there are $\left(\frac{1000 \mathrm{~m}}{50 \mathrm{~m}}\right)+1=21$ pairs of OV MV BPL topologies for given original OV MV BPL topology.

Except for the database properties regarding the number of the considered OV MV BPL topologies, the second step for the creation of the detailed MLFLM database urges the database enrichment with information concerning coupling reflection coefficients. In order to simplify the procedure and apply the findings of [22], a set of database specifications is here given:

- $k_{\text {sect,min }}$ is the lower monotonic section bound, which is assumed to be equal to 1 in this paper, and $k_{\text {sect,max }}$ is the upper monotonic section bound, which is assumed to be equal to 20 in this paper

- The operation frequency range and the flat fading subchannel frequency spacing are assumed equal to $1-30 \mathrm{MHz}$ and $1 \mathrm{MHz}$, respectively. Therefore, the number of subchannels $u$ in the examined frequency range is equal to 30 .

- Arbitrarily, the $\mathrm{WtG}^{3}$ coupling scheme is applied during the following simulations. As it is usually done [9], [27], [28], [29], [18], [30], the selection of representative coupling schemes is a typical procedure for the sake of reducing manuscript size and simplicity.

- For each OV MV BPL topology that is considered in the database, the two cases of termination loads should be considered (either short- or open-circuit). 
In accordance with the previous assumptions and eqs (5) and (6) of [23], there is a need for inserting $2 \times 20=40$ approximated theoretical OV MV BPL coupling reflection coefficient column vectors (say, $\overline{\overline{\Gamma_{\text {theor, } 1}^{\mathrm{WtG}}}}$ and $\overline{\overline{\Gamma_{\text {theor, } 2}^{\mathrm{WtG}}}}$ ) per each possible OV MV BPL topology pair of the database, which corresponds to the respective 20 monotonic sections. Each approximated theoretical OV MV BPL coupling reflection coefficient column vector $\overline{\overline{\Gamma_{\text {thtG }}^{\mathrm{Wtg}, k}}}, k=1 \ldots 2$ consists of 30 elements (say, $\overline{\overline{\Gamma_{\text {theor }, k}^{\mathrm{WtG}}}}\left(f_{i}\right), k=1 \ldots 2, i=1, \ldots, 30$ ), which corresponds to the respective 30 measurement frequencies $f_{i}, i=1, \ldots, 30$. Therefore, the detailed OV MV BPL topology database comprises $40 \times 30=1200$ elements for each OV MV BPL topology pair. Since main distribution line faults can be treated as either short- or open-circuit terminations, there are $2 \times 600=1200$ element insertions per pair or $50^{\prime} 400$ insertions in total that should be added into the database concerning its enrichment with coupling reflection coefficient attributes.

Let assume that a main distribution line fault occurs and the measured coupling reflection coefficients from the side of the transmitting and receiving end of the original OV MV BPL topology are equal to $\overline{\Gamma_{\text {meas }, 1}^{\mathrm{WtG}}}$ and $\overline{\Gamma_{\text {meas }, 2}^{\mathrm{WtG}}}$, respectively. Note that $\overline{\Gamma_{\text {meas }, 1}^{\mathrm{WtG}}}$ and $\overline{\Gamma_{\text {meas. } 2}^{\mathrm{WtG}}}$ are the coupling reflection coefficients of the modified OV MV BPL topologies when the nature of the termination load is considered. For the 30 measurement frequencies $f_{i}, i=1, \ldots, 30$, the measured OV MV BPL reflection coefficient column vectors $\overline{\Gamma_{\text {meas, } 1}^{\mathrm{WtG}}}$ and $\overline{\Gamma_{\text {meas }, 2}^{\mathrm{WtG}}}$ consist of the respective measured coupling reflection coefficients from the transmitting end $\overline{\Gamma_{\text {meas }, 1}^{\mathrm{WtG}}}\left(f_{i}\right), i=1, \ldots, 30$ and receiving end $\overline{\Gamma_{\text {meas }, 2}^{\mathrm{WtG}}}\left(f_{i}\right), i=1, \ldots, 30$. L1PMA software processes its inputs, which are the measured coupling reflection coefficient column vectors $\overline{\Gamma_{\text {meas }, k}^{\mathrm{WtG}}}, k=1 \ldots 2$, and gives as outputs the respective approximated measured OV MV BPL reflection coefficient column vectors $\overline{\overline{\Gamma_{\text {meas }, k}^{\mathrm{WtG}}}}, k=1 \ldots 2$.

The third and final step of MLFLM is the localization of the main distribution line faults through the computation of MDLFI $_{\text {par }}$ and MDLFI. Similarly to MDLFI of [23], $\mathrm{MDLFI}_{k}, k=1 \ldots 2$ of the respective two modified OV MV BPL topologies is given by

$$
M D L F I_{k}=\frac{\sum_{k_{\mathrm{sect}}=1}^{20} M D L F I_{\mathrm{par}, k}\left(k_{\mathrm{sect}}\right)}{600}, k=1 \ldots 2
$$

where

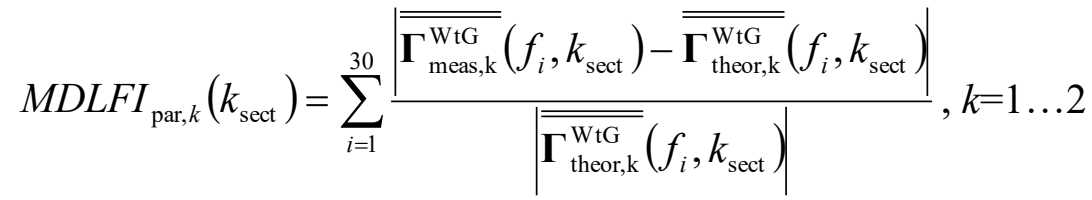

Observing eq. (1), several interesting remarks concerning the application of MDLFI to localize main distribution line faults can be pointed out: 
- In order to exploit the efficiency of MDLFI to identify main distribution line faults that are closer to the measurement instrument, $M D L F I_{1}$ and $\mathrm{MDLFI}_{2}$ offer a differential advantage through the two available measurement locations of coupling reflection coefficients (i.e., the side of the transmitting and receiving end of the original OV MV BPL topology). With reference to eq. (1), if the main distribution line fault is located near to the transmitting end then MDLFI presents higher values than the respective ones of $\mathrm{MDLFI}_{2}$. In the reverse case of a main distribution line fault located near to the receiving end, $\mathrm{MDLFI}_{2}$ presents a more reliable identification behavior in comparison with $\mathrm{MDLFI}_{1}$.

- According to [23], MDLFI describes the relative error between L1PMA approximations of the measured and theoretical data. The identification of the main distribution line faults has been achieved through the comparison of MDLFI with certain critical thresholds that mainly depends on the modified OV MV BPL topologies. Here, MLFLM exploits the differential reception of $\mathrm{MDLFI}_{1}$ and $\mathrm{MDLFI}_{2}$ by comparing the measured coupling reflection coefficients with the respective ones of all the available pairs of MLFLM database. The pairs of MLFLM database that present approximately the minimum MDLFI 1 and $\mathrm{MDLFI}_{2}$ are the candidate modified OV MV BPL topologies while the location of the main distribution line fault and the nature of the terminal loads can straightforward been found from the insertion number of the MLFLM database.

- MLFLM performance is based on the detection of the MDLFI pair in the MLFLM database. Since MDLFI depends on: (i) the original OV MV BPL topology; (ii) the nature of the terminal load; (iii) the location of main distribution line faults; and (iv) the intensity of measurement differences, so do MDLFI 1 and $\mathrm{MDLFI}_{2}$, the immunity performance of MLFLM against aforementioned MDLFI dependencies to localize the main distribution line faults through $\mathrm{MDLFI}_{1}$ and $\mathrm{MDLFI}_{2}$ is assessed in Sec.4.

\section{Numerical Results and Discussion}

\subsection{Simulation Goals and Parameters}

The four original OV MV BPL topologies of Sec. 2 are simulated with the purpose of accurately localizing potential main distribution line faults by applying MLFLM. In this Section, the MLFLM efficiency is assessed when various factors such as the complicity of the original OV MV BPL topology, the nature of the terminal load and the location of main distribution line faults are assumed. Similarly to the identification of main distribution line faults, the localization of main distribution line faults becomes a challenging issue when real-life conditions are simulated through the application of measurement differences. For that reason, the measurement differences that occur in $\mathrm{OV}$ MV BPL networks during the determination of coupling reflection coefficients are typically described by continuous uniform distributions (CUD) with range from 0 to a maximum CUD value that is equal to $\alpha_{\mathrm{MD}}$. It is evident that the measurement difference CUD remains the same during the simultaneous coupling reflection coefficient measurements from the transmitting and receiving end of the original OV MV BPL topology.

The combined impact of measurement differences and main distribution line faults on the OV MV BPL coupling reflection coefficient is here investigated. First, three different measurement difference CUDs are assumed, namely: 
(i) CUD with $a_{\mathrm{MD}}=0$ (no measurement differences are assumed while this CUD case is denoted as CUD case A); (ii) CUD with $a_{\mathrm{MD}}=0.1$ (denoted as CUD case B); and (iii) CUD with $a_{\mathrm{MD}}=0.2$ (denoted as CUD case C). Second, three different locations of main distribution line faults are applied, namely: (a) fault location situated at $250 \mathrm{~m}$ from the transmitting end (denoted as Fault case A); (b) fault location situated at 500m from the transmitting end (denoted as Fault case B); and (c) fault location situated at $750 \mathrm{~m}$ from the transmitting end (denoted as Fault case C).

\subsection{Fault Localization of Main Distribution Line Faults by Applying MLFLM (General Case)}

Let assume that the fault case $\mathrm{A}$ has been identified in accordance with [23] during the operation of the urban case and the fault localization begins through the initiation of MLFLM procedures. Since the main distribution line fault occurs, $\overline{\Gamma_{\text {meas, } 1}^{\mathrm{WtG}}}$ and

$\overline{\Gamma_{\text {meas }, 2}^{\mathrm{WtG}}}$ are available through the coupling reflection coefficient measurements from the transmitting and receiving end side, respectively. Since the MLFLM database has already been theoretically implemented for the urban case for all the available pairs of modified OV MV BPL topologies, MDLFIs (i.e., $\mathrm{MDLFI}_{1}$ and $\mathrm{MDLFI}_{2}$ ) are determined for each pair of the aforementioned modified OV MV BPL topologies. Therefore, in Fig. 1, MDLFIs (i.e., MDLFI $_{1}$ and $\mathrm{MDLFI}_{2}$ ) are plotted versus the distance from the transmitting end of the original topology when CUD case A is adopted and the terminal load is assumed to be short-circuit during the main distribution line fault case A. For the sake of simplicity, only the pairs of the modified OV MV BPL topologies with shortcircuit termination loads are retrieved from the MLFLM database. In Fig. 2, same plots are drawn with Fig. 1 but for the case of an open-circuit terminal load while the MLFLM database pairs of modified OV MV BPL topologies with open-circuit termination loads are only examined.

From Figs. 1 and 2, it is obvious that MDLFIs both receive their minimum values when the fault location from the transmitting end, which is related with the MLFLM database, is equal to the actual distance of the main distribution line fault from the transmitting end. Since fault case A mandates that the main distribution line fault is situated at $250 \mathrm{~m}$ from the transmitting end, $\mathrm{MDLFI}_{1}$ and $\mathrm{MDLFI}_{2}$ are both equal to 0 in

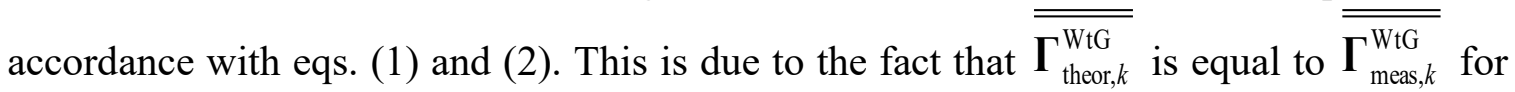
given $k$ since no measurement differences are considered in the CUD case A. In all the other cases, MDLFIs receive values that are greater than zero. However, this ideal situation of localizing the main distribution line faults through the zeroing of MDLFIs stands only in the case of no measurement differences and, hence, the case of the inclusion of measurement differences requires further examination (see the following subsection).

\subsection{The Impact of the Measurement Differences on the Fault Localization by Applying MLFLM}

Although the fault localization through the simultaneous minimization of MDLFIs can be considered as an intuitive method to accurately localize main distribution 


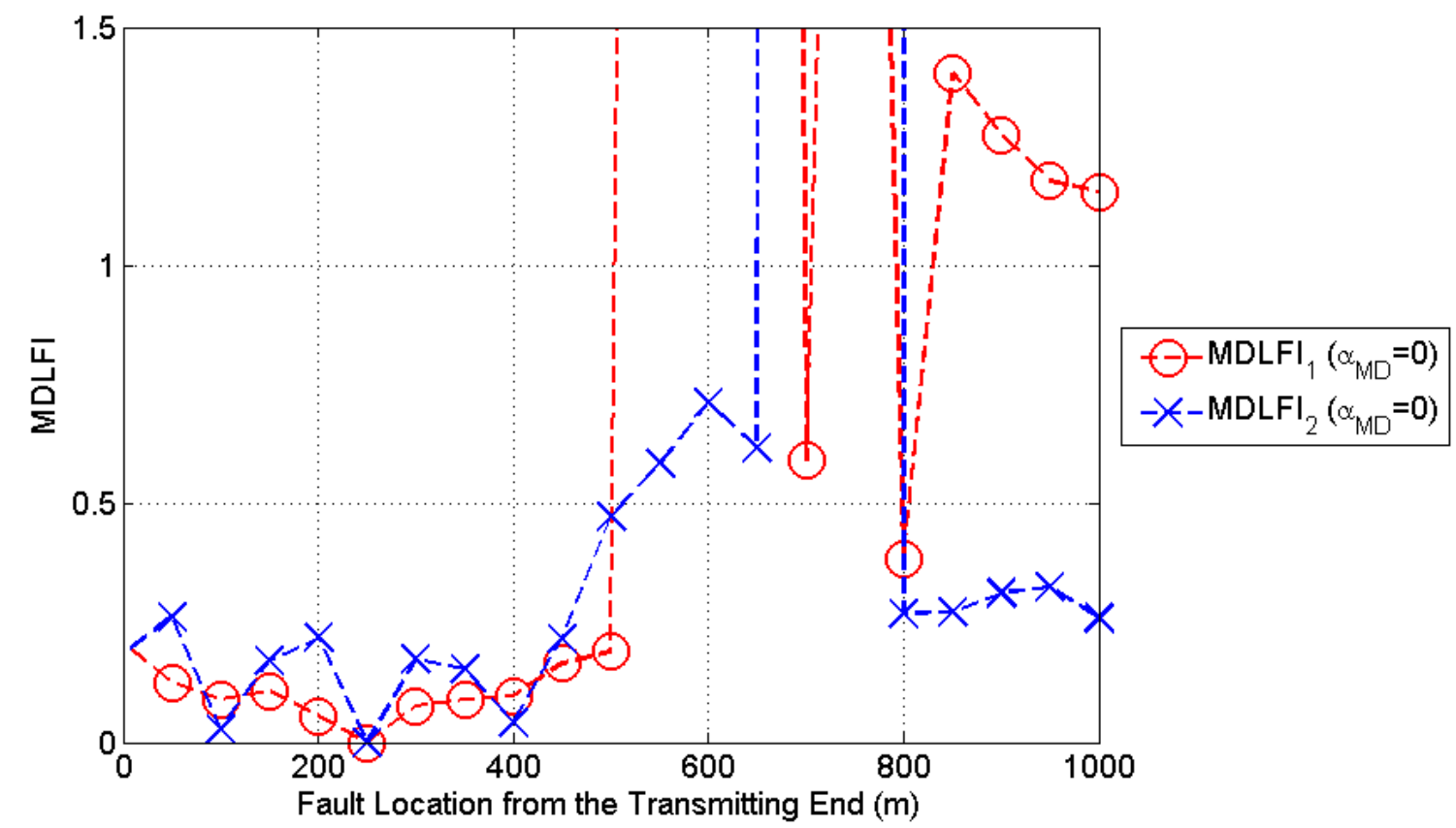

Figure 1. MDLFIs of the urban OV MV BPL topology versus the fault location from the transmitting end when fault case A (i.e., main distribution line fault situated at $250 \mathrm{~m}$ from the transmitting end) and CUD case A (i.e., $a_{M D}=0$ ) are assumed (the terminal load is assumed to be a short-circuit termination load).

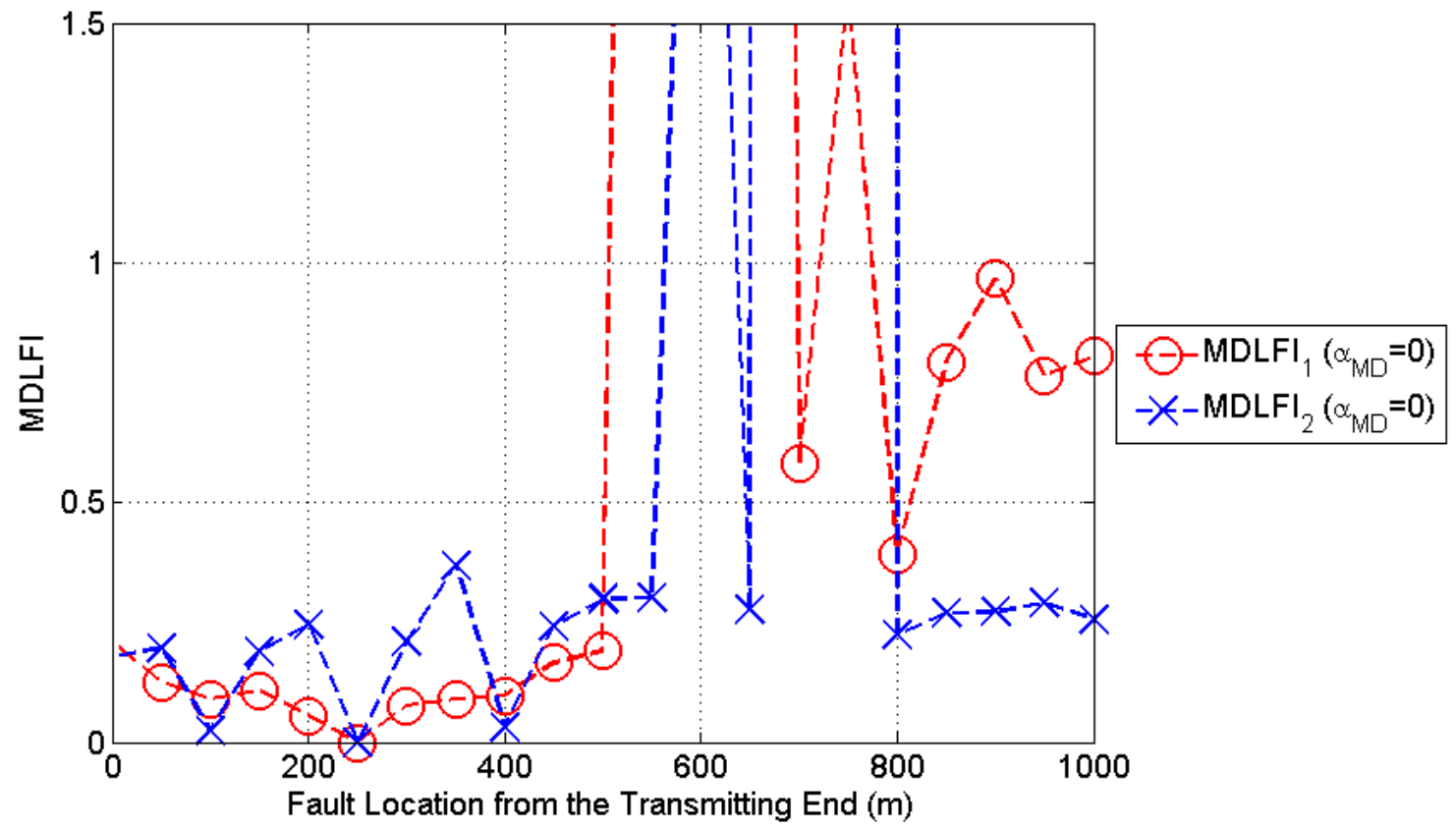

Figure 2. Same curves with Figure 1 but for the case of open-circuit terminal load.

line faults, this has verified until now only during the cases of no measurement differences. However, this finding needs to be validated during the fault localization by applying the simultaneous minimization of MDLFIs when measurement differences of various CUD magnitudes occur. 
Similarly to the subsection 4.2, in Fig. 3, MDLFIs are plotted versus the distance from the transmitting end when the terminal load is assumed to be short-circuit during the main distribution line fault case A for CUD case A, B and C. In Fig. 4, same plots are drawn with Fig. 3 but for the case of an open-circuit terminal load.

Observing Figs. 3 and 4, it is again verified that MDLFIs can accurately localize the main distribution line faults through their simultaneous minimization even if measurement differences of various magnitudes occur. Actually, the localization of main distribution line faults becomes easier when measurement difference magnitudes remain low since then both MDLFIs tend to zero. When measurement difference magnitudes increase, MDLFIs start to differ from zero even at the location of the main distribution line fault but still maintaining lower values than ones of the other locations. However, MDLFI behavior strongly depends on the location of the main distribution line fault as verified in [5], [23]. Hence, the validity of the previous findings needs to be examined for various fault cases in the following subsection.

From the Figs. 3 and 4, it should also be noted that MDLFIs can efficiently localize main distribution line faults regardless of the nature of the terminal load (i.e., either short- or open-circuit termination). This is an interesting remark that allows the examination of only one case (say, the case of short-circuit terminal load) to be considered in the rest of this paper.

\subsection{The Impact of the Main Distribution Line Fault Location on the Fault Localization by Applying MLFLM}

As been presented in subsections 4.2 and 4.3, MDLFIs have very efficiently determined the location of the main distribution line fault during the fault case A. In fact, the location of the main distribution line fault has been retrieved by the simultaneous minimization of MDLFIs even if measurement differences occur during the presence of a main distribution line fault. In fact, MDLFI metric acts as a dissimilarity metric resembling with the metrics of relative and absolute error but in a more sophisticated manner since it exploits the inherent piecewise monotonic trend of coupling transfer functions and coupling reflection coefficients of OV MV BPL networks [25], [26], [24]. Since MDLFI acts as a dissimilarity metric between the coupling transfer functions of original and modified OV MV BPL topologies [5], [23], MDLFI achieves to more easily identify main distribution line faults that are located near to the transmitting end than to receiving end since the differences between theoretical coupling reflection coefficients of the original OV MV BPL topology and measured coupling reflection coefficients of the measured OV MV BPL topology remains significant. In order to cope with this finding of [23], coupling reflection coefficient measurements are received both from the transmitting and receiving end by applying $\mathrm{MDLFI}_{1}$ and $\mathrm{MDLFI}_{2}$, respectively, as determined in eqs. (1) and (2).

Indeed, in Fig. 5, MDLFIs are plotted versus the distance from the transmitting end when the terminal load is assumed to be short-circuit during the main distribution line fault for all the CUD cases examined so far. Note that the Fault case B is here assumed. In Fig. 6, same plots are drawn with Fig. 5 but for the Fault case C. 


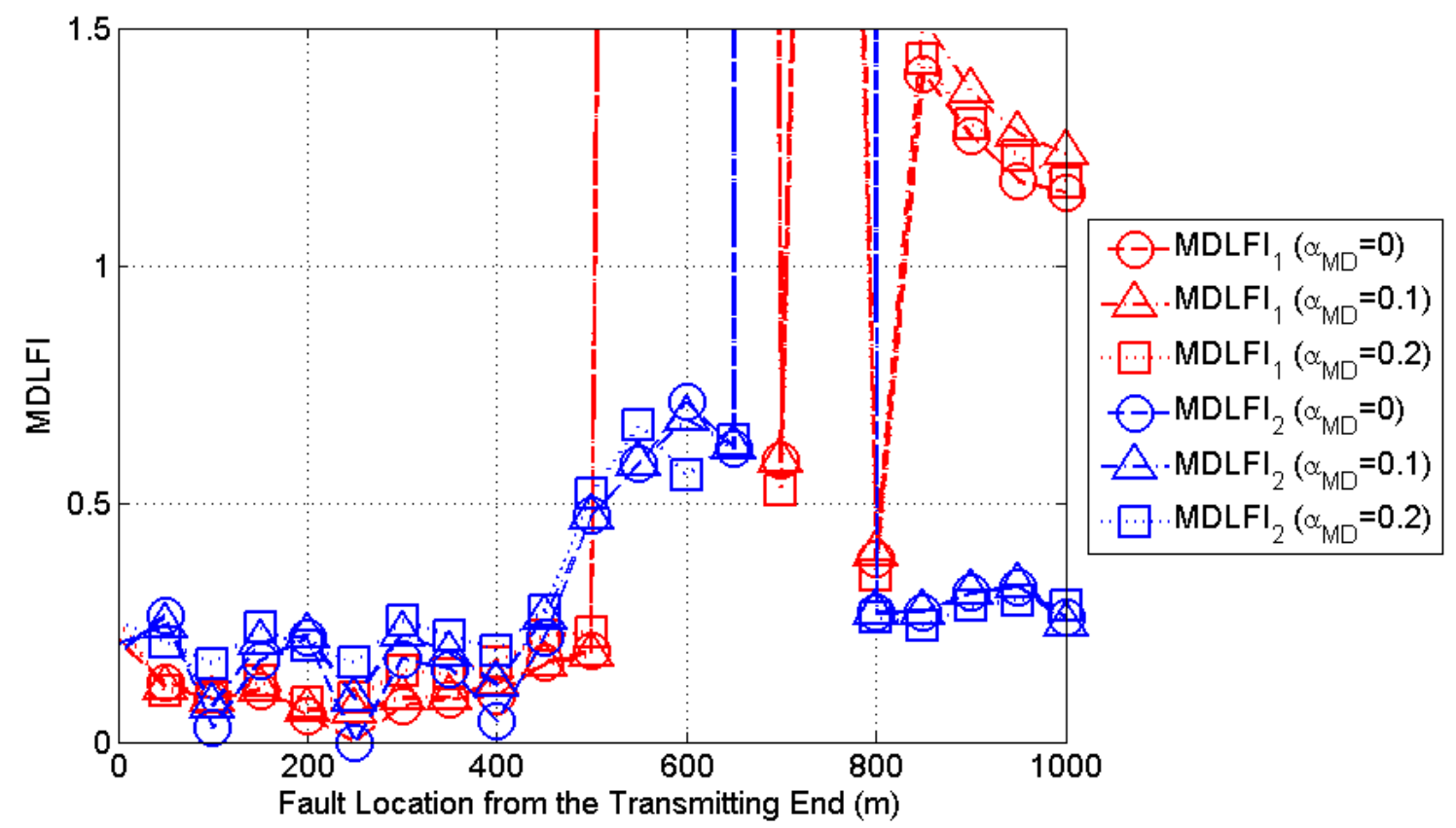

Figure 3. MDLFIs of the urban OV MV BPL topology versus the fault location from the transmitting end for various CUD magnitudes when fault case $A$ is applied (the terminal load is assumed to be a short-circuit termination load).

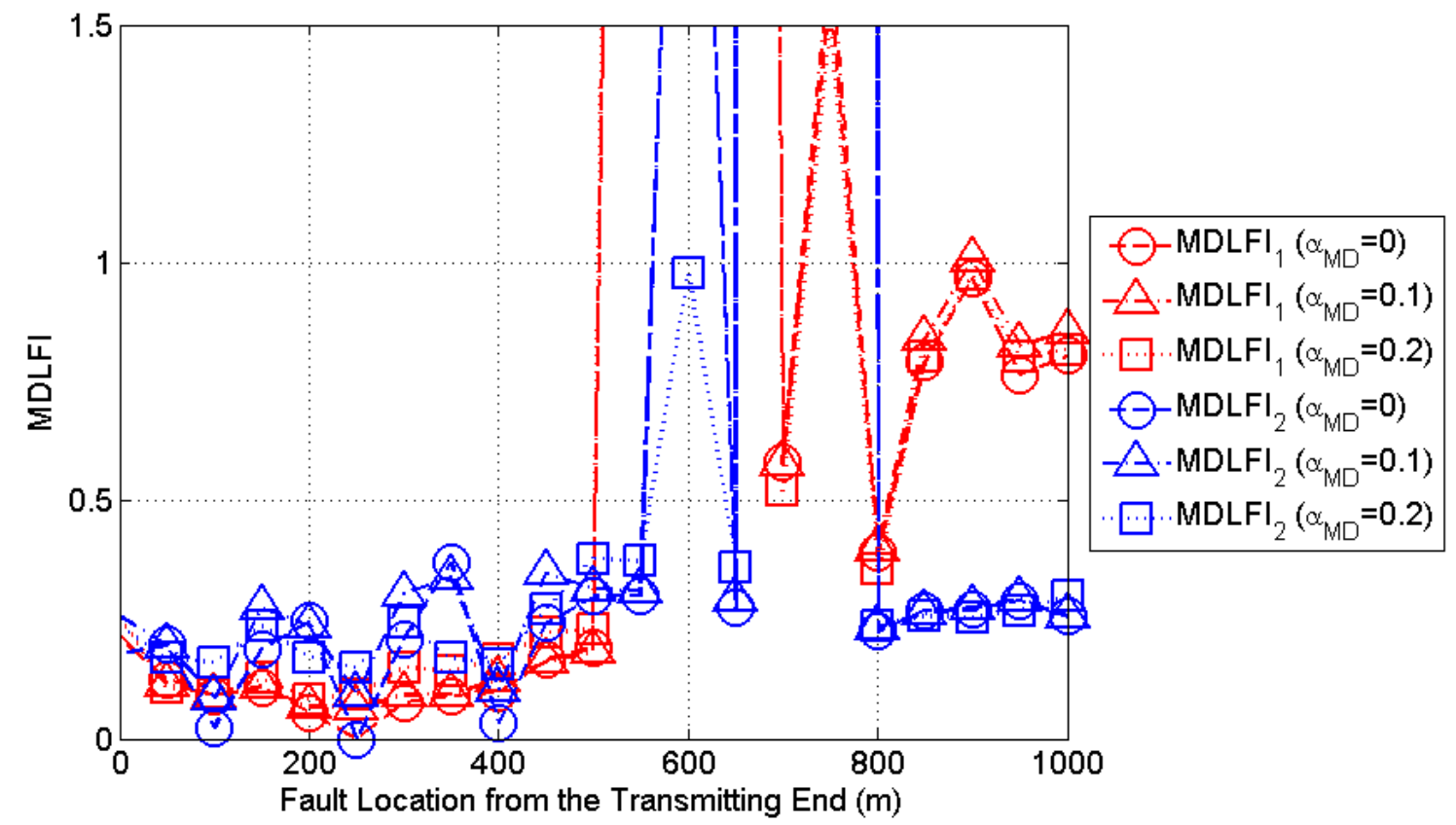

Figure 4. Same curves with Figure 3 but for the case of open-circuit terminal load. 


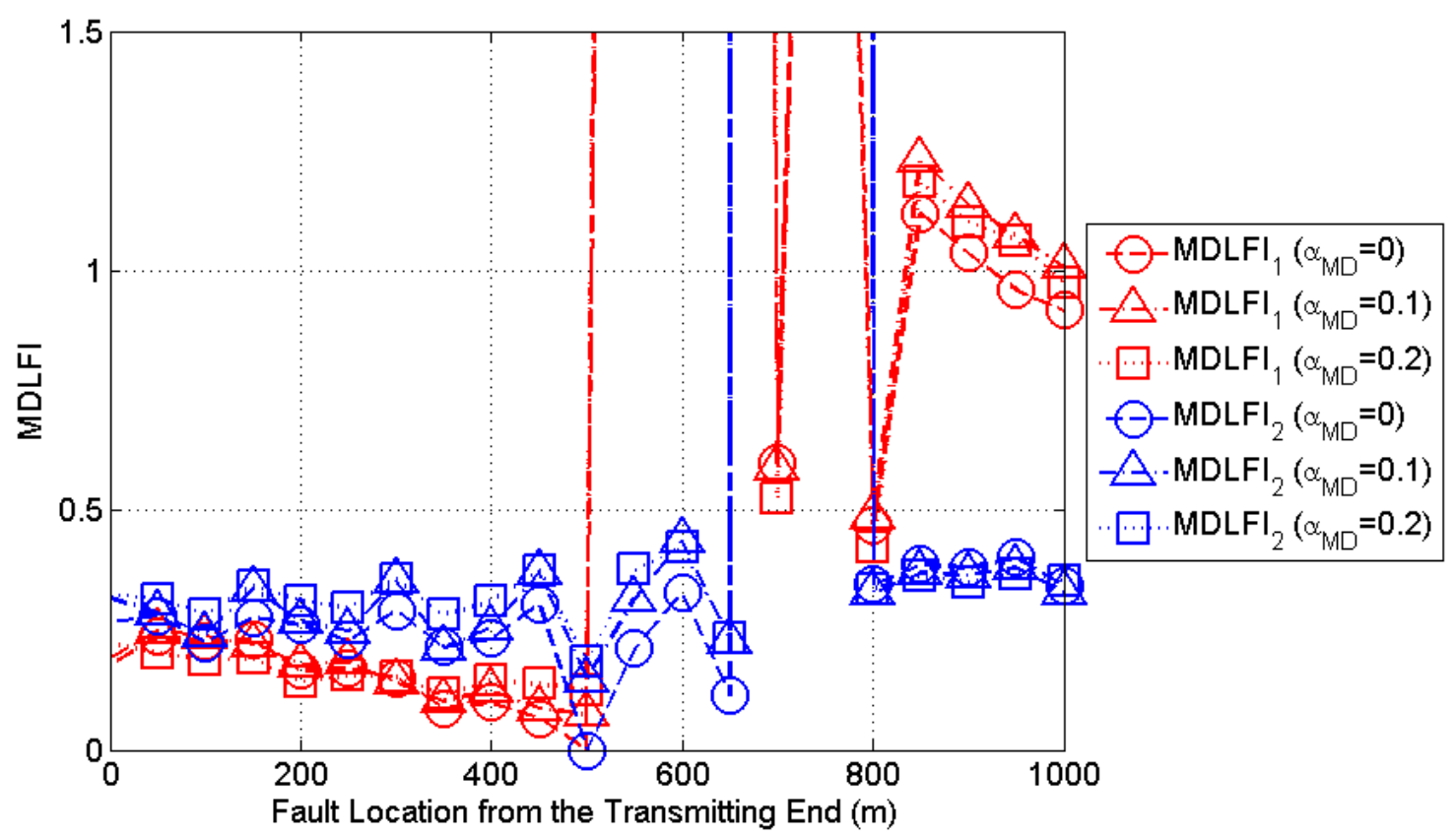

Figure 5. MDLFIs of the urban OV MV BPL topology versus the fault location from the transmitting end for various CUD cases when Fault case B occurs (the terminal load is assumed to be a short-circuit termination load).

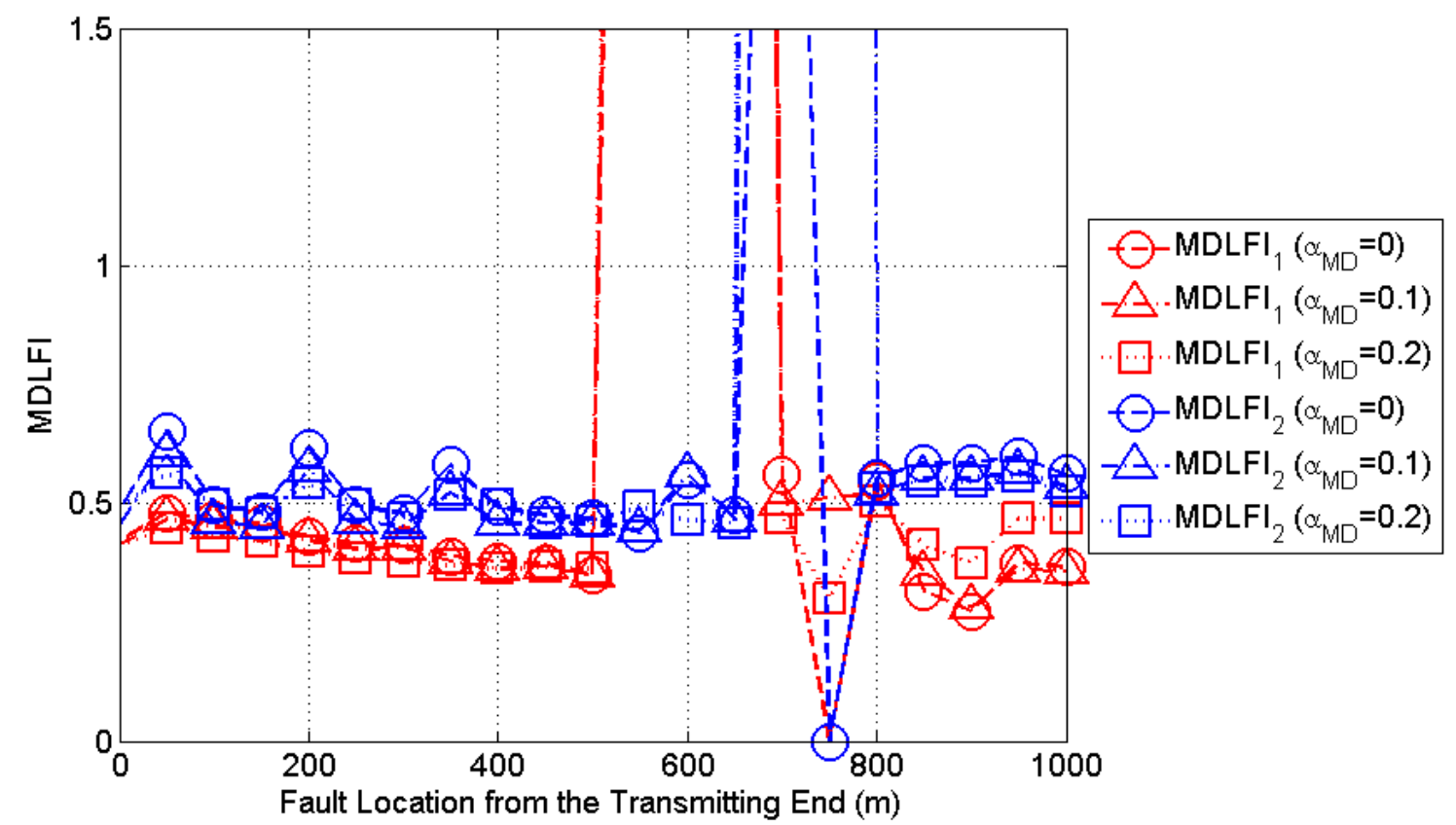

Figure 6. Same curves with Figure 5 but for the Fault case $\mathrm{C}$.

Comparing Figs. 3, 5 and 6, the combined use of the available MDLFIs can allow the exact localization of main distribution line faults regardless of their location across the distribution grid. Also, the MLFLM localization of main distribution line faults remains robust even in the cases of normal measurement differences. In fact, the combined operation of $\mathrm{MDLFI}_{1}$ and $\mathrm{MDLFI}_{2}$ achieves to bypass the inherent difficulty of dissimilarity metrics, which is the easier identification of main distribution line faults 
near to the end that are applied using the respective coupling reflection coefficient measurements. Thus, the combined minimization of MDLFIs that is the core assumption of MLFLM methodology successfully copes with the different locations of main distribution line faults.

However, until now, the performance of the localization of main distribution line faults by applying MLFLM has focused on the performance assessment of the localization in the urban OV MV BPL topology when various factors affecting the methodology performance has been taken into account. In the following subsection, the performance of MLFLM methodology is assessed for the other three indicative original OV MV BPL topologies described in Sec. 2 -say, suburban, rural and "LOS" OV MV BPL topologies-.

\subsection{The Impact of the Original OV MV BPL Topologies on the Fault Localization by Applying MLFLM}

Previously been mentioned, the presence of main distribution line faults across the original OV MV BPL topologies has as a result the definition of two new modified OV MV BPL topologies which have the termination load of the main distribution line fault as the only common point. Since there are two different measurement points, which are located at the transmitting and receiving end of the original OV MV BPL topology, these two modified OV MV BPL topologies operate as the complimentary topologies of the original OV MV BPL topology. Until now, only the urban OV MV BPL topology of Sec. 2, had been used as the original OV MV BPL topology where the main distribution line faults have been imposed. In this subsection, the MLFLM efficiency against different original OV MV BPL topologies is assessed.

Indeed, in Fig. 7, MDLFIs are plotted versus the distance from the transmitting end of the original suburban OV MV BPL topology when the terminal load is assumed to be short-circuit. Note here that the Fault case A and all the available CUD cases are assumed in Fig. 7. In Figs. 8 and 9, same plots are drawn with Fig. 7 but for the rural and "LOS" cases, respectively. 


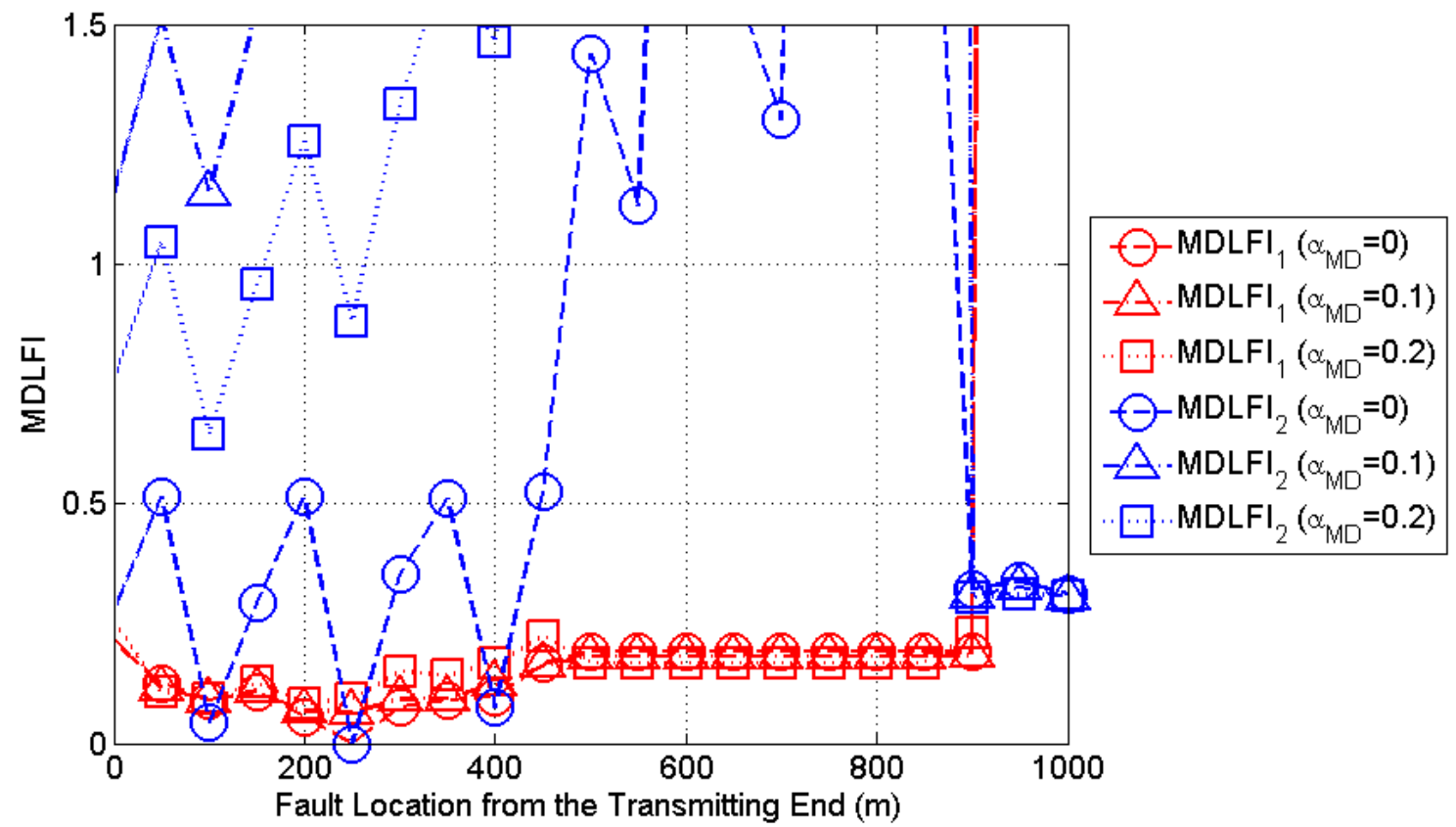

Figure 7. MDLFIs of the original suburban OV MV BPL topology versus the fault location from the transmitting end for various CUD cases when Fault case A occurs (the terminal load is assumed to be a short-circuit termination load).

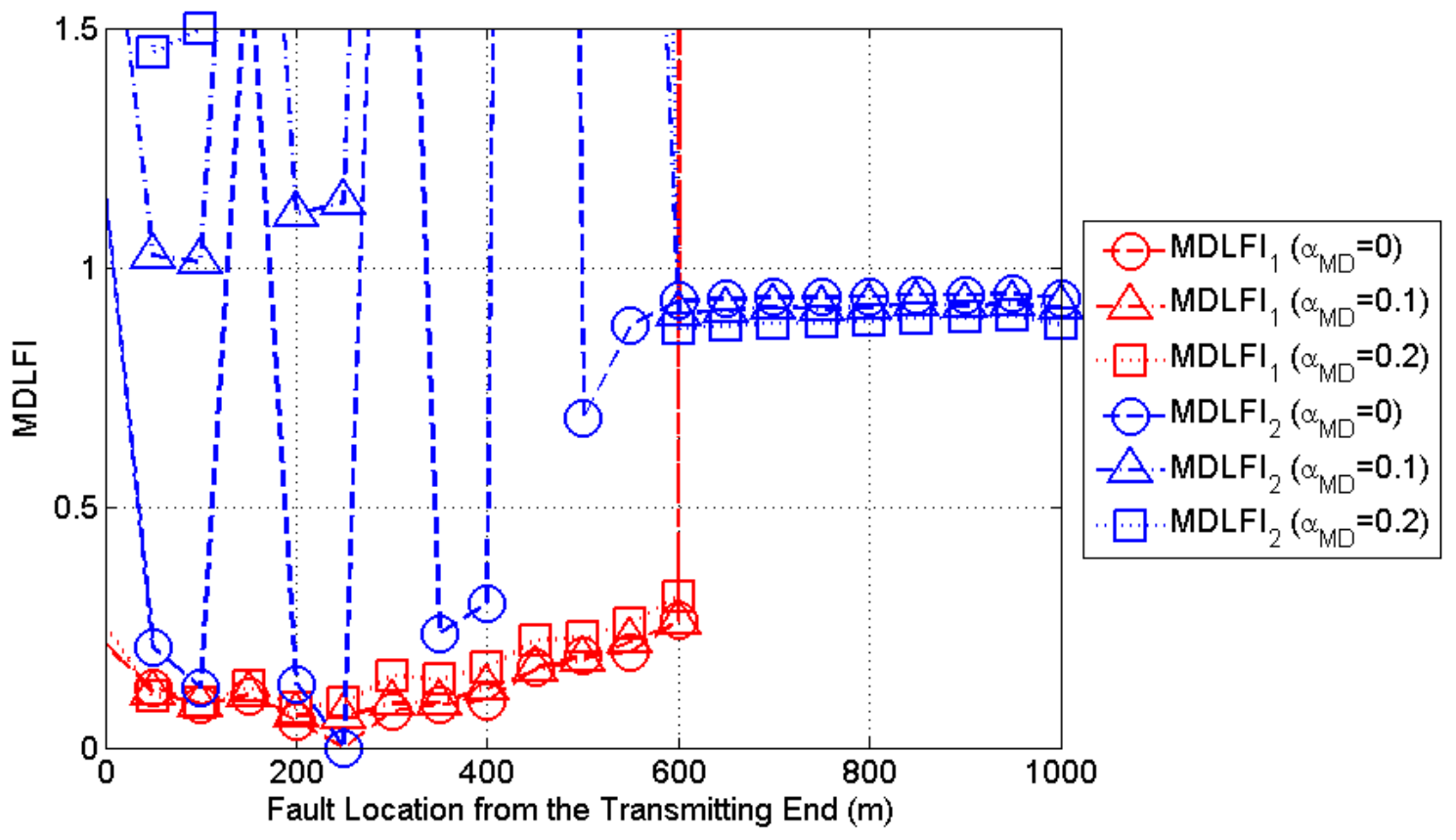

Figure 8. Same curves with Figure 7 but for the rural case. 


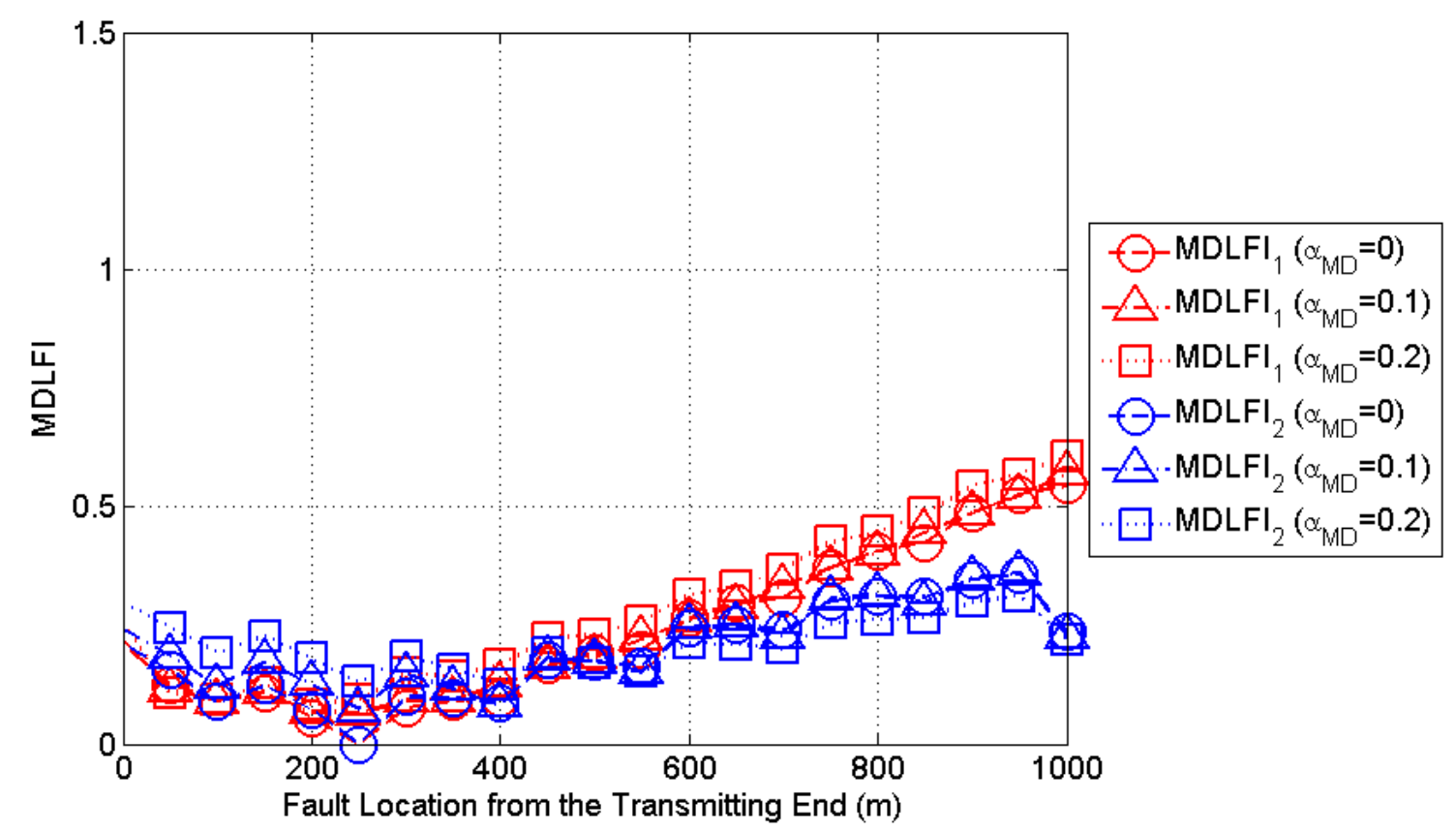

Figure 9. Same curves with Figure 7 but for the "LOS" case.

Comparing Figs. 3, 7, 8 and 9, MDLFIs can successfully localize main distribution line faults regardless of the intensity of the occurred measurement differences and the original OV MV BPL topology. More specifically, when the CUD magnitude of measurement differences is equal to zero MDLFIs are also equal to zero in all the examined original OV MV BPL topologies. When the CUD magnitude of measurement differences increases, MDLFIs take value greater than zero but their trend and their minima still imply the exact location of the examined main distribution line fault. Since the simultaneous minimization of MDLFIs is required in order to securely localize the main distribution line faults, this allows MLFLM to crosscheck the fault location even if the identification by applying one of MDLFIs remains low but does not take the minimum value. As presented, the latter case is rare and can stand only in the cases of intense measurement differences.

In this paper, the exact localization of main distribution line faults has been achieved by applying MLFLM when the identification of the presence of main distribution line faults is assured as described in [5], [23]. Actually, MLFLM is based on the application of MDLFIs by taking advantage of the double coupling reflection coefficient measurement sets (i.e., the first set from the transmitting end side and the other one from the receiving end side of the original OV MV BPL topology) and the MLFLM database. Then, the simultaneous minimization of MDLFIs offers the exact location of the main distribution line fault across the original OV MV BPL topology regardless of the CUD magnitude of measurement differences, the location of the main distribution line fault and the original OV MV BPL topology. 


\subsection{General Remarks Concerning the Identification and Localization of Faults} and Instabilities across Distribution Power Grids

Already been identified in [3], [22], [24]-[26], various serious problematic conditions can occur across the distribution power grid such as faults and instabilities. More specifically, faults and instabilities can further be divided into two subcategories each. On the basis of a typical OV MV BPL topology, which is presented in Fig. 10(a), the subcategories of faults and instabilities are reported as follows:

- Faults: All the interruptions that can occur across the lines of a distribution power grid. The two fault subcategories of line interruptions are: (i) Fault in main distribution line -see $\quad$ Fig. 10(b)-; and (ii) Fault in branch line -see Fig. 10(c)-.

- Instabilities: All the failures that can occur in the equipment across the distribution power grid. There are two subcategories of equipment failures, namely: (iii) Instability in branch interconnections -see Fig. 10(d)-; and (iv) Instability in branch terminations -see Fig. 10(e)-. 


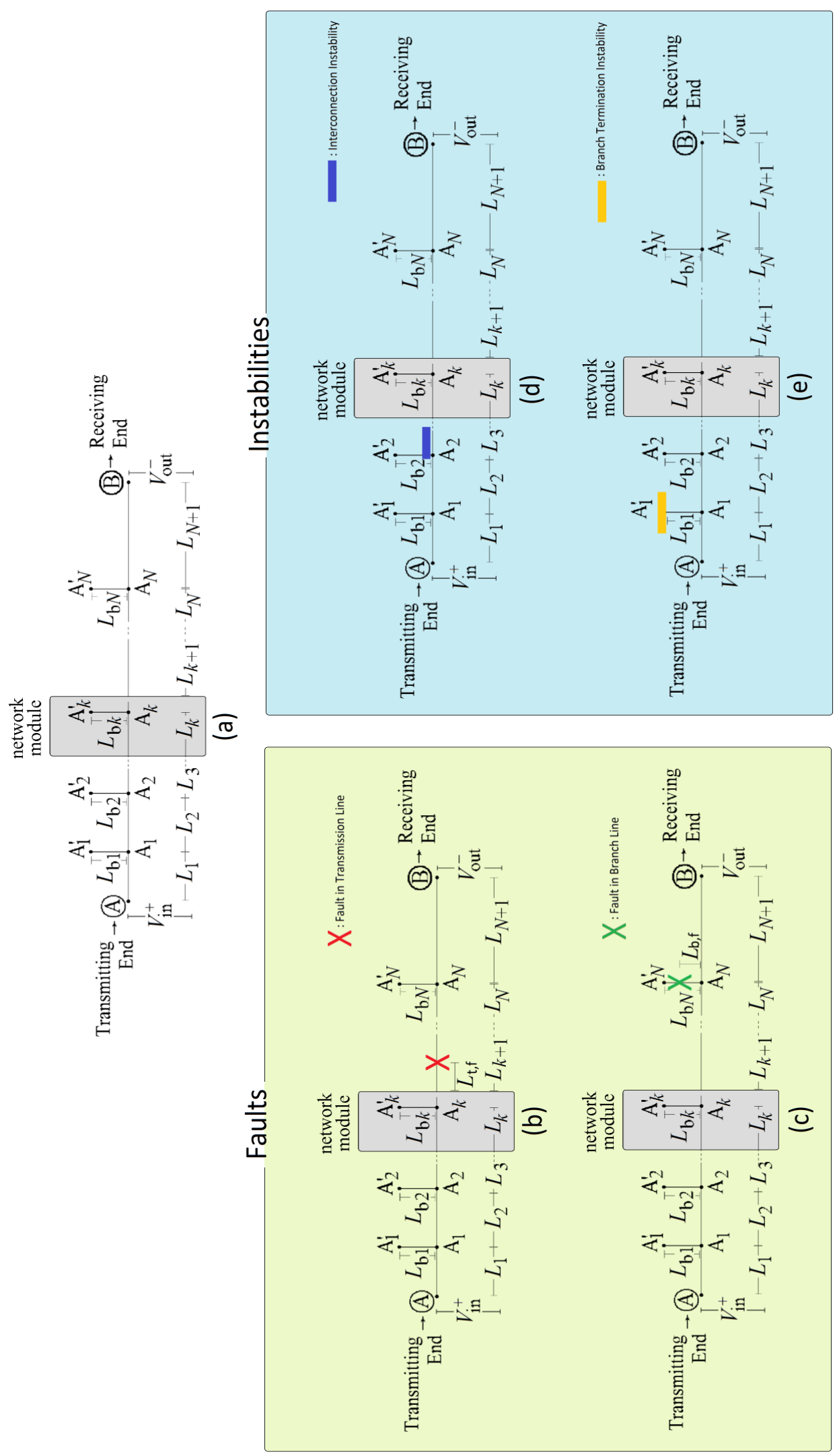

Figure 10. (a) General OV MV BPL topology [22], [24]. (b, c) Faults in OV MV BPL topologies. (d, e) Instabilities in OV MV BPL topologies [3]. 
Until now, the identification and localization methodology of the fault in branch line, the instability in branch interconnections and the instability in branch terminations have well defined and analyzed in [3], [22], [24]-[26]. However, the case of the identification and localization of the main distribution line faults had been treated till these three papers as a reductio ad absurdum case. This set of three papers has achieved to cover this identification / localization methodology gap through the proposal and application of MLFLM methodology by identifying and exactly localizing possible main distribution line faults [5], [23]. Therefore, a complete methodology of identifying and localizing possible faults and instabilities across distribution power grids is now available.

\section{Conclusions}

In this paper, which is the last part of a set of three manuscripts, the detailed presentation and the performance assessment of MLFLM have been demonstrated. In fact, MLFLM describes the identification and localization methodology of main distribution line faults across the distribution power grids. This paper concludes the study of faults and instabilities across transmission and distribution power grids revealing the strong potential of the BPL technology in order to identify and exactly localize potential failures across the grids.

This paper has first reported the required steps to create MLFLM database, which is the essential part of MLFLM. Second, MDLFIs, which are the accompanied metrics of MLFLM, and their application to the coupling reflection coefficient insertions of MLFLM database have been proposed. Third, the interaction procedure between MLFLM database and MDLFIs that finally leads to the localization of the main distribution line faults has been outlined.

After MLFLM theoretical analysis, various scenarios concerning the localization of main distribution line faults have been investigated by applying MLFLM. More specifically, MLFLM has successfully localized the occurred main distribution line faults regardless of the examined OV MV BPL topologies, the location of the main distribution line faults and the CUD magnitude of the measurement differences. Among the main conclusions of MLFLM application, some of them that deserve special attention are: (i) the same MLFLM efficiency of localizing main distribution line faults despite the complexity of the examined original OV MV BPL topology; (ii) through the combined measurement operation of coupling reflection coefficients from the transmitting and receiving end, MDLFIs have been simultaneously defined, thus rendering MLFLM immune against the location of main distribution line faults; and (iii) MLFLM can mitigate measurement differences since the combined minimization of MDLFIs secures MLFLM performance.

After this set of papers, apart from the identification and localization methodology of the fault in branch line, the instability in branch interconnections and the instability in branch terminations, the case of the identification and localization of the main distribution line faults has also been achieved. Now, a complete methodology of identifying and localizing possible faults and instabilities across transmission and distribution power grids, which is based on the signal transmission theory and signal processing techniques through informatics, is now available. 


\section{CONFLICTS OF INTEREST}

The author declares that there is no conflict of interests regarding the publication of this paper.

\section{References}

[1] L. Lutz, A. M. Tonello, and T. G. Swart, Power Line Communications: Principles, Standards and Applications from multimedia to smart grid, John Wiley \& Sons, 2016.

[2] G. Artale, A. Cataliotti, V. Cosentino, D. Di Cara, R. Fiorelli, S. Guaiana, and G. Tine, "A New Low Cost Coupling System for Power Line Communication on Medium Voltage Smart grids," IEEE Trans. on Smart Grid, to be published, 2017.

[3] A. G. Lazaropoulos, "Measurement Differences, Faults and Instabilities in Intelligent Energy Systems - Part 2: Fault and Instability Prediction in Overhead High-Voltage Broadband over Power Lines Networks by Applying Fault and Instability Identification Methodology (FIIM)," Trends in Renewable Energy, vol. 2, no. 3, pp. 113 - 142, Oct. 2016. [Online]. Available: http://futureenergysp.com/index.php/tre/article/view/27/33

[4] A. G. Lazaropoulos, A. Sarafi, and P. G. Cottis, "The emerging smart grid - A pilot MV/BPL network installed at Lavrion, Greece," in Proc. Workshop on Applications for Powerline Communications WSPLC 2008, Thessaloniki, Greece, Oct. 2008.2 [Online]. Available: http://newton.ee.auth.gr/WSPLC08/Abstracts\%5CSG 3.pdf

[5] A. G. Lazaropoulos, "Main Line Fault Localization Methodology in Smart Grid Part 1: Extended TM2 Method for the Overhead Medium-Voltage Broadband over Power Lines Networks Case," Trends in Renewable Energy, vol. 3, no. 3, pp. 2-25, 2017.

[6] A. G. Lazaropoulos, "Factors Influencing Broadband Transmission Characteristics of Underground Low-Voltage Distribution Networks," IET Commun., vol. 6, no. 17, pp. 2886-2893, Nov. 2012.

[7] A. G. Lazaropoulos and P. G. Cottis, "Transmission characteristics of overhead medium voltage power line communication channels," IEEE Trans. Power Del., vol. 24, no. 3, pp. 1164-1173, Jul. 2009.

[8] A. G. Lazaropoulos and P. G. Cottis, "Capacity of overhead medium voltage power line communication channels," IEEE Trans. Power Del., vol. 25, no. 2, pp. 723-733, Apr. 2010.

[9] A. G. Lazaropoulos and P. G. Cottis, "Broadband transmission via underground medium-voltage power lines-Part I: transmission characteristics," IEEE Trans. Power Del., vol. 25, no. 4, pp. 2414-2424, Oct. 2010.

[10] A. G. Lazaropoulos and P. G. Cottis, "Broadband transmission via underground medium-voltage power lines-Part II: capacity," IEEE Trans. Power Del., vol. 25, no. 4, pp. 2425-2434, Oct. 2010.

[11] A. G. Lazaropoulos, "Broadband transmission characteristics of overhead highvoltage power line communication channels," Progress in Electromagnetics Research B, vol. 36, pp. 373-398, 2012. [Online]. Available: http://www.jpier.org/PIERB/pierb36/19.11091408.pdf 
[12] A. G. Lazaropoulos, "Towards broadband over power lines systems integration: Transmission characteristics of underground low-voltage distribution power lines," Progress in Electromagnetics Research B, 39, pp. 89-114, 2012. [Online]. Available: http://www.jpier.org/PIERB/pierb39/05.12012409.pdf

[13] A. G. Lazaropoulos, "Broadband transmission and statistical performance properties of overhead high-voltage transmission networks,"Hindawi Journal of Computer Networks and Commun., 2012, article ID 875632, 2012. [Online]. Available: http://www.hindawi.com/journals/jcnc/aip/875632/

[14] A. G. Lazaropoulos, "Towards modal integration of overhead and underground low-voltage and medium-voltage power line communication channels in the smart grid landscape: model expansion, broadband signal transmission characteristics, and statistical performance metrics (Invited Paper)," ISRN Signal Processing, vol. 2012, Article ID 121628, 17 pages, 2012. [Online]. Available: http://www.isrn.com/journals/sp/aip/121628/

[15] A. G. Lazaropoulos, "Review and Progress towards the Common Broadband Management of High-Voltage Transmission Grids: Model Expansion and Comparative Modal Analysis," ISRN Electronics, vol. 2012, Article ID 935286, pp. $1-18,2012 . \quad$ [Online]. Available: http://www.hindawi.com/isrn/electronics/2012/935286/

[16] A. G. Lazaropoulos, "Review and Progress towards the Capacity Boost of Overhead and Underground Medium-Voltage and Low-Voltage Broadband over Power Lines Networks: Cooperative Communications through Two- and ThreeHop Repeater Systems," ISRN Electronics, vol. 2013, Article ID 472190, pp. 1-19, 2013. [Online]. Available: http://www.hindawi.com/isrn/electronics/aip/472190/

[17] A. G. Lazaropoulos, "Green Overhead and Underground Multiple-Input MultipleOutput Medium Voltage Broadband over Power Lines Networks: EnergyEfficient Power Control," Springer Journal of Global Optimization, vol. 2012 / Print ISSN 0925-5001, pp. 1-28, Oct. 2012.

[18] P. Amirshahi and M. Kavehrad, "High-frequency characteristics of overhead multiconductor power lines for broadband communications," IEEE J. Sel. Areas Commun., vol. 24, no. 7, pp. 1292-1303, Jul. 2006.

[19] T. Sartenaer, "Multiuser communications over frequency selective wired channels and applications to the powerline access network" Ph.D. dissertation, Univ. Catholique Louvain, Louvain-la-Neuve, Belgium, Sep. 2004. [Online\} Available: https://dial.uclouvain.be/pr/boreal/en/object/boreal\%3A5010/datastream/PDF 12/ view

[20] T. Calliacoudas and F. Issa, "“"Multiconductor transmission lines and cables solver," An efficient simulation tool for plc channel networks development," presented at the IEEE Int. Conf. Power Line Communications and Its Applications, Athens, Greece, Mar. 2002.

[21] A. G. Lazaropoulos, "Best L1 Piecewise Monotonic Data Approximation in Overhead and Underground Medium-Voltage and Low-Voltage Broadband over Power Lines Networks: Theoretical and Practical Transfer Function Determination," Hindawi Journal of Computational Engineering, vol. 2016, Article ID 6762390, 24 pages, 2016. doi:10.1155/2016/6762390. [Online]. Available: https://www.hindawi.com/journals/jcengi/2016/6762390/cta/

[22] A. G. Lazaropoulos, "Measurement Differences, Faults and Instabilities in Intelligent Energy Systems - Part 1: Identification of Overhead High-Voltage 
Broadband over Power Lines Network Topologies by Applying Topology Identification Methodology (TIM)," Trends in Renewable Energy, vol. 2, no. 3, pp. 85 - 112, Oct. 2016.

[23] A. G. Lazaropoulos, "Main Line Fault Localization Methodology in Smart Grid Part 2: Extended TM2 Method, Measurement Differences and L1 Piecewise Monotonic Data Approximation for the Overhead Medium-Voltage Broadband over Power Lines Networks Case," Trends in Renewable Energy, vol. 3, no. 3, pp. 26-61, 2017.

[24] A. G. Lazaropoulos, "Improvement of Power Systems Stability by Applying Topology Identification Methodology (TIM) and Fault and Instability Identification Methodology (FIIM) - Study of the Overhead Medium-Voltage Broadband over Power Lines (OV MV BPL) Networks Case," Trends in Renewable Energy, vol. 3, no. 2, pp. 102 - 128, Apr. 2017. [Online]. Available: http://futureenergysp.com/index.php/tre/article/view/34/pdf

[25] A. G. Lazaropoulos, "Power Systems Stability through Piecewise Monotonic Data Approximations - Part 1: Comparative Benchmarking of L1PMA, L2WPMA and L2CXCV in Overhead Medium-Voltage Broadband over Power Lines Networks," Trends in Renewable Energy, vol. 3, no. 1, pp. 2 - 32, Jan. 2017. [Online]. Available: http://futureenergysp.com/index.php/tre/article/view/29/34

[26] A. G. Lazaropoulos, "Power Systems Stability through Piecewise Monotonic Data Approximations - Part 2: Adaptive Number of Monotonic Sections and Performance of L1PMA, L2WPMA and L2CXCV in Overhead Medium-Voltage Broadband over Power Lines Networks," Trends in Renewable Energy, vol. 3, no. 1, pp. $33-60$, Jan. 2017. [Online]. Available: http://futureenergysp.com/index.php/tre/article/view/30/35

[27] A. Canova, N. Benvenuto, and P. Bisaglia, "Receivers for MIMO-PLC channels: Throughput comparison," in Proc. IEEE Int. Symp. Power Line Communications and Its Applications, Rio de Janeiro, Brazil, Mar. 2010, pp. 114-119.

[28] D. Schneider, J. Speidel, L. Stadelmeier, and D. Schill, "Precoded spatial multiplexing MIMO for inhome power line communications," in Proc. IEEE Global Telecommunications Conference, New Orleans, LA, USA, Nov./Dec. 2008, pp. 1-5.

[29] M. Zimmermann and K. Dostert, "Analysis and modeling of impulsive noise in broad-band powerline communications," IEEE Trans. Electromagn. Compat., vol. 44, no. 1, pp. 249-258, Feb. 2002.

[30] T. Esmailian, F. R. Kschischang, and P. G. Gulak, "In-building power lines as high-speed communication channels: Channel characterization and a test channel ensemble," Int. J. Commun. Syst., vol. 16, pp. 381-400, May 2003.

Article copyright: (C) 2017 Athanasios G. Lazaropoulos. This is an open access article distributed under the terms of the Creative Commons Attribution 4.0 International License, which permits unrestricted use and distribution provided the original author and source are credited. 